\title{
FaceID-Bones Narrate: A Semi Automated Approach for Forensic Facial Reconstruction
}

\author{
Anuradha K. Madugalla $^{\# 1}$, Roshan N. Rajapakse ${ }^{\# 2}$,Ishari .U. Amarasinghe ${ }^{\# 3}$, Vinavi H. Padmathilake ${ }^{\# 4}$, Anuja T. \\ Dharmaratne $^{\# 5}$,DamithaSandaruwan ${ }^{\# 6}$, MudithaVidanapathirana ${ }^{* 7}$

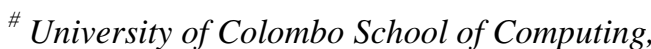 \\ 35, Reid Avenue, Colombo 07, Sri Lanka \\ ${ }^{1}$ anu.mdg@gmail.com \\ ${ }^{2}$ rosh474@gmail.com \\ 3 ishari1987@gmail.com \\ ${ }^{4}$ padmathilakevinavi@gmail.com \\ 5 atd@ucsc.cmb.ac.lk \\ ${ }^{6} \mathrm{dsr} @$ ucsc.cmb.ac.lk \\ *Department of Forensic Medicine, Faculty of Medical Sciences, University of Sri Jayewardenepura, Sri Lanka \\ ${ }^{7}$ mudithavidana@yahoo.co.uk
}

\begin{abstract}
Forensic facial reconstruction is still at its infancy in Sri Lanka and is yet to utilize the advanced technologies of other countries. Hence introducing a more efficient multimedia based technique to the local forensic officials in order to improve the efficiency and the accuracy of the reconstructions is the aim of this study. In contrast to the other mechanisms used for facial reconstruction by others, this paper adopts a novel approach of muscle based facial reconstruction which goes hand in hand with the manual reconstruction process. The adopted process involved, acquiring a 3D model of the skull and digitally sculpting muscles in a 3D environment, followed by adding different facial features to improve identification. The research also encompassed a tissue thickness analysis that is conducted for the first time on Sri Lankans as well as a facial component analysis, both of which were needed to improve the accuracy of the final output. This procedure was attempted on cases of the age category 20-30 and of medium weight. The outputs and the process were evaluated with different parties such as general public, forensic officials, lawyers and CID all of which are to be benefited from this application. The ultimate goal of conducting the study was to understand and overcome the challenges faced in developing this novel application for the Sri Lankan Forensic officials and to establish the first unit for facial reconstruction in Sri Lanka.
\end{abstract}

Keywords - forensic facial reconstruction, tissue thickness, facial components, digital sculpting, Sri Lankan, muscle-anatomy

\section{INTRODUCTION}

Upon the occurrence of a naturalor amanmade disaster,such as terrorist bombings, mob uprisings, Tsunami, etc...the administrative bodies of a country has to handle piles of unidentified human? corpses. And when murder victims plunged deep underground are surfaced years later, even then the administrative bodies have to handle unidentifiable corpses. In these situations when neither their personal effects nor body tissues can define the identity of the deceased, the investigations are moved on to the facial reconstruction stage. Yet it has to be borne in mind that this stage is also known as the facial approximation, because the end result of this process, is a face that would only hold some resemblance to the deceased, and will not be the exact face of him/her. Once an approximate is reconstructed, it is circulated via digital and paper mediums with hope of a citizen being able to identify it. Upon such identification, the stated identification is verified using DNA, fingerprint or dental records analysis. Hence facial reconstruction is of great use to criminal investigators.
But apart from criminal investigators, facial reconstruction is also used by biological anthropologists in approximating the look of early hominid forms, as well as by archaeologists in verifying the remains of historic figures.

In spite of all these uses, Sri Lanka currently does not adopt any facial reconstruction method. The authors of the paper intended to solve this shortcoming by recommending a manual, 2D or 3D reconstruction method. However, around the world, the facial reconstruction process is conducted mostly via manual mechanisms or 3D automated mechanisms. Out of the two mechanisms, the manual method has significant disadvantages such as being highly time consuming and requiring expensive resources and human expertise. In contrast to it, 3D reconstruction is morecost and time effective. Therefore, the authors considered that introducing a computer aided 3D reconstruction method to the Sri Lankan Forensic Scientists would be the best solution.

\section{Week 1 Week 2}

12345671234567

\section{Manual \\ Reconstruction}

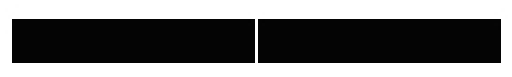

\section{D \\ Reconstruction}

Fig.1Time taken for the manual and 3D techniques for facial reconstruction

A solution for implementing the suggested 3D facial reconstruction in Sri Lanka was developed by the authors in collaboration with the Department of Forensic Sciences in Colombo and Sri Jayewardenepura Universities. One of the leading experts of facial reconstruction in Sri Lanka has assisted the researchers in this study and he is intending to implement the first centre for facial reconstruction in Sri Lanka with the developed solution.

The feasibility of implementing such a solution, its effectiveness and the challenges in implementing and developing the application are discussed in this paper. The impact of the application upon the society is observed through the feedback gathered from different analysis and the feasibility of the novel semi-automated method is also evaluated through these analyses. 


\section{RELATED WORK}

Forensic facial reconstruction has been active in the field of forensics from the latter part of the 18th century and it has been practised using various methodologies over the years. Although the earliest attempts at "reconstruction" were at a very trivial level, they have aided in bringing the practice to what it is today.

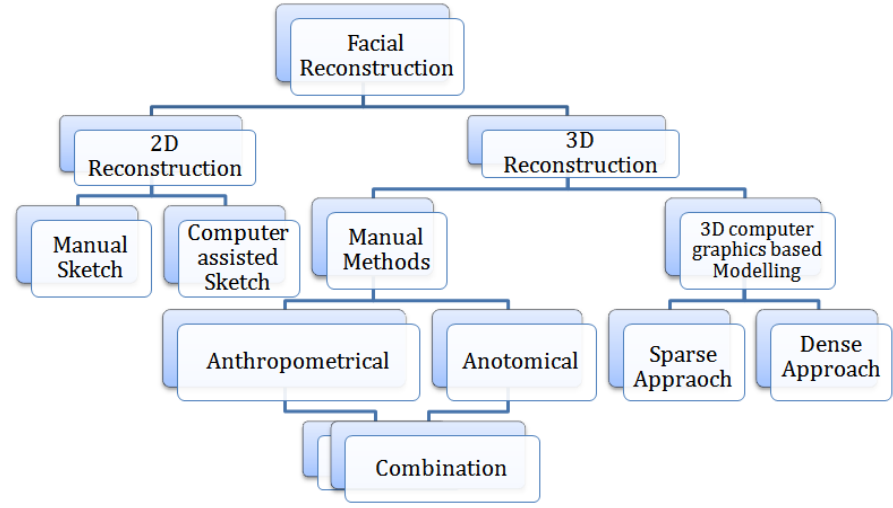

Fig. 2.Types of facial reconstruction

Historically, two broad categories of facial reconstruction can be established from literature, 2D \& 3D (Figure 2). However, when chronologically ordered, the manual techniques of reconstruction of both the 2D and 3D methods took place in early years the reconstruction.

The very first attempts of trying to use skeletal remains to reconstruct the face, although not following a scientific procedure dates back to $6750-6250 \mathrm{BC}$ where skulls were used to model the face (Pre-Pottery Neolithic B PPNB period) [1]. Other efforts of creating the actual facial appearance of the deceased came in the form of "Death Masks" (Figure 3). It is noted that in 18th century this technique was used for studies related to physiognomic features in important persons and notorious criminals and to identify missing persons[2].

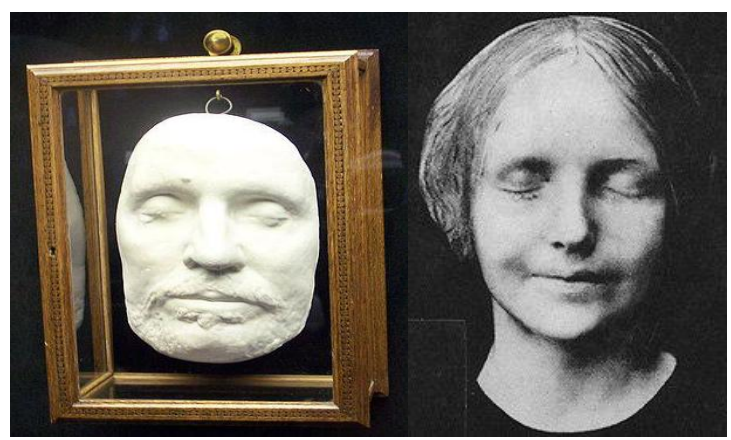

Fig. 3 Reconstruction via Death Masks [a. Oliver Cromwell; b.L'Inconnue de la Seine] [3], [4]

The Scientific approach to facial reconstruction initiated in the late 19th century where cadavers were used to measure facial tissue thickness[5]. It is noted in the literature that the reconstruction of the face of the popular composer Johann Sebastian Bach was done by the German anatomist on the year of 1895 [6] and this is considered the first process that followed a scientific approach. He used a set of soft-tissue measurements obtained from White German cadavers to construct a three dimensional clay model of Bach. This method of reconstruction involves creating a plasticine model of the skull and sculpting the face by using clay on top of the model. What follows are the main variations of this method.

\section{Anatomical (Russian) method}

Prominence is given to facial musculature in the reconstruction

2. Anthropometrical (American) method

The main basis of this method is to gather tissue thickness data initially and reconstruct the face using that information

3. Combination methods (often referred to as British or Manchester method) [6]

This approach is based on rebuilding each individual facial muscle by using tissue thickness depth markers as base and covering the clay copy of dry skull with appropriate muscles (Figure 4) instead of simply filling the soft tissues' depth.
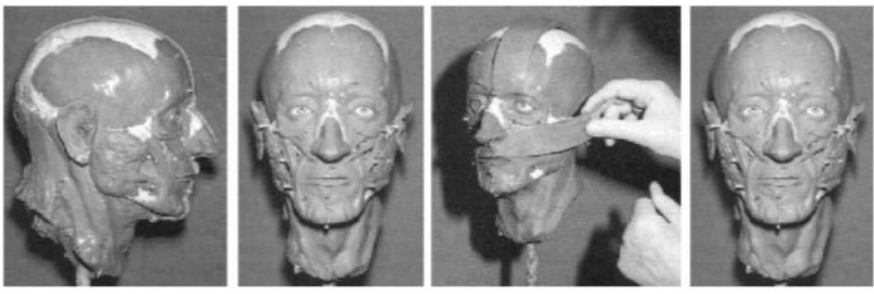

Fig.4Facial reconstruction manual technique[7]

However, the human effort and the specific clay and plasticine materials required for reconstruction were costly and the process involved several man hours.

The other category of reconstruction is the 2D methodology. The main outputs of this process are two dimensional facial profile drawings and it said to be substantially faster than the manual way of reconstruction[8]. Most of these techniques are based on drawing contours of X-rays of the deceased person's skull thus resulting in only a 2D output. And it is mentioned that the technique is not accurate since many cranial structures are not visible or at the correct scale. Therefore it requires the collaboration of an artist and a forensic anthropologist

Abate A.F et al.[7]stated that this is the simplest reconstructive technique, based on the positioning of markers showing soft tissues (tissue depth? Or tissues' depth ) depths on the frontal and side images of a given skull. Then a contour fitting the markers and the skull itself is traced on a partially transparent sheet. This curve acts as a reference for the subsequent drawing phase which involves the anatomic and anthropological knowledge of the identikit artist and leads to a graphic reconstruction of a compatible face (Figure 5)
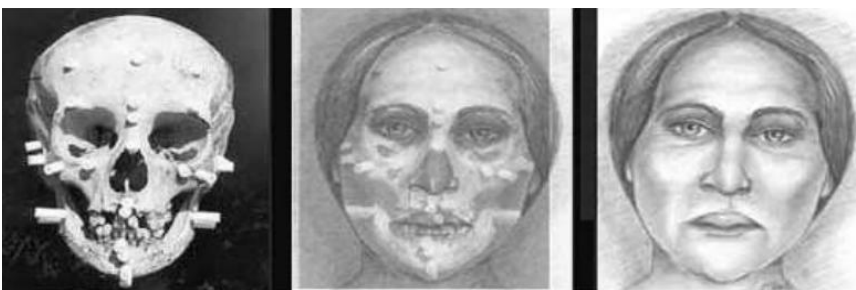

Fig. 5Markers applied to a skull copy, contour fitting the markers [7]

The most effective means of generating facial approximations in today's context is to use 3D computer images/Graphics based methods. This method has similarities to the manual sculpting technique but with greater effectiveness and flexibility.

The focus of this study, computer aided 3D reconstruction, has been carried out by different researchers in different ways and each of them has had their own advantages as well as 
disadvantages. However, each of them had to initiate the process by first obtaining the 3D model of the skull. Computed Tomography (CT) scanning techniques and a sequence of photographic skull image frames [9] were used by some to generate the 3D model of the skull. In the more modern researches sophisticated techniques such as Facial Optical Surface Scanners [10],Electro spatial digitizers[5], Cyberwave TM Model Color -3D Scanheadscanners [11]have been used.

Once a 3D model was acquired, then it was prepared for the skin building process. According to literature this was done using two main techniques.

1. Dense Approach

2. Sparse Approach

The dense approach used deformation algorithms where a reference skull model is deformed to match the measurements of the unidentified skull model (a volumetric approach)[12]

In this method developed by Nelson and Michael, a database is created with several 3D skull models belonging to different age/gender groups. Out of the database the reference skull models with similar age/gender range to the unidentified skull are selected. The selected skull models and the subjects' skull are marked with a set of control points. Based on these control points the reference skull with the closest matching spatial distribution of control points is selected. The reference skull is then deformed based on the same control points.[13]
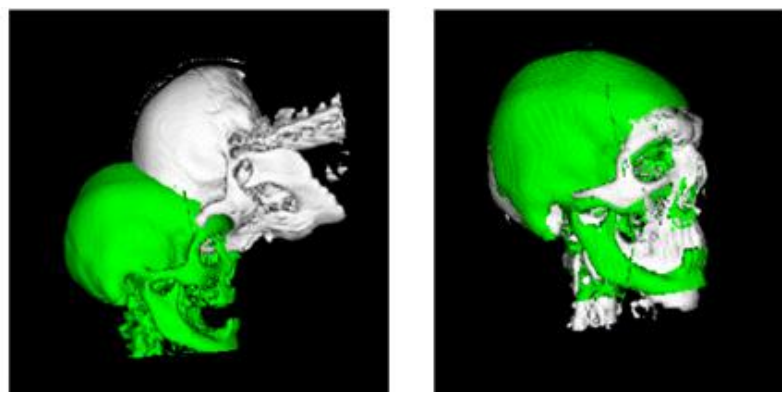

Fig. 6Aligning the two Skulls: Green skull is the reference model and the white skull is the Subjects' model. The left image shows an initial misalignment and the right image shows the two skulls after the alignment [12]

The second and in fact the most vastly used technique in facial reconstruction studies; the sparse approach, used tissue depth land markers. These landmarks were positioned based on the anthropological data (via CT and MRI head scans) and the number of landmarks to be placed was subjected to change based on the study.

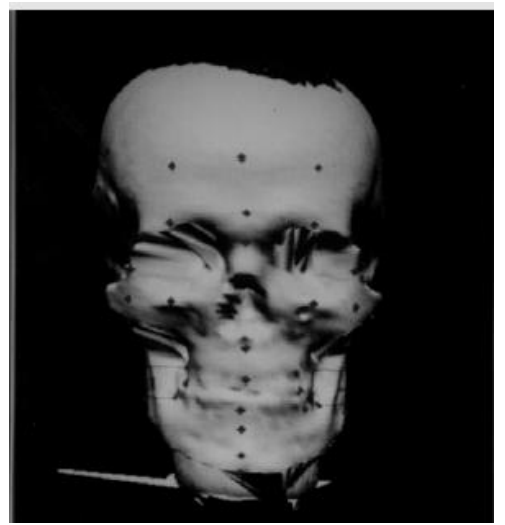

Fig. 7 Skull with landmarks in position [10]

In the sparse method, after placing the landmarks on the skull, the subsequent steps that have to be adopted to complete the reconstruction again has differed based on the research groups and studies.
One such was the method presented by the Professor of Forensic Medical Sciences, Peter Vanezis. He had presented the technique of fitting a facial template to the skull and adjusting that template according to the markers[10]. These templates were stored in databases, categorized based on the skull they were supposed to fit into, and in the reconstruction process the artist had chosen a template that had standard average features that matched best with the subjects' skull anthropologically. This method had been adopted by some other researchers as well, and in all these studies, the templates had been collected by taking head scans of a large number of people, as seen in [7] and [10]. But in later studies, it was felt that this approach resulted in a reconstructed face that had a high resemblance to the donors face (templates face)[14], [15]. This was felt to be a major shortcoming since an ideal reconstruction was expected to result in a reconstructed face that had a resemblance to the deceased and not to the donor. Hence a change in the approach was supported by the forensic community.It resulted in a shift from template based reconstruction on to skull as the sole basis reconstruction approach.

In the research done by Davy et al[15]rather than using reference images from a database, the skull was used as the sole base and NURBS curves were used to create elliptical cross sections which were then used as the base of the face.

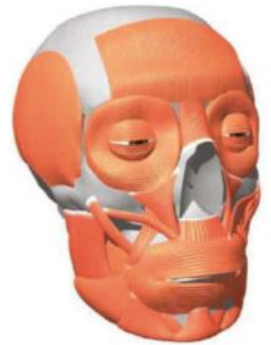

Fig. 8Facial reconstruction manual technique[15]

Another study that usedthe skull as sole basis approach, has utilized 3dsmax and the software's inbuilt scripting functionality for reconstruction. They have used mesh calculations to produce the reconstructed face. [8](Figure 9)
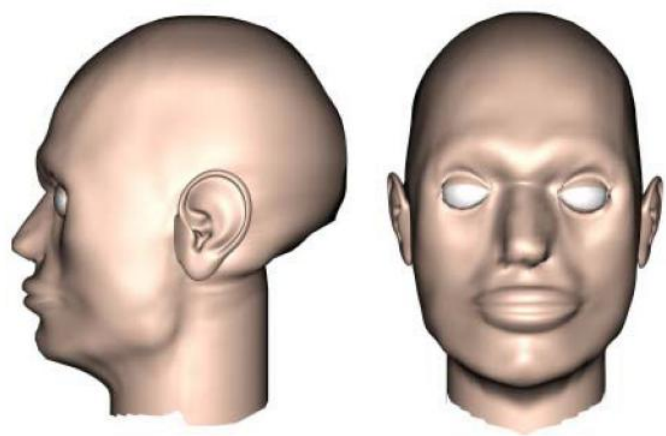

Fig. 9Reconstructed face of the research of [8]

Facial features such as eyes, eyebrows, nose and lips were added as the final step. In most of the previous studies the facial features of living humans were acquired by digitizing faces of the subject and then placing those facial components on the reconstructed face [16]

Although several studies point out that adding facial features when no actual information is available would increase the possibility of identification, it is argued by some that additional facial features should be kept as minimum as possible due to the reason that additional features would mislead the identification. 


\section{METHODOLOGY}

The developed solution involved a framework with a set of processes and tools. This framework is described as phases in this third section of the paper, and in each phase the tools and techniques that were used for the process are described.

Test cases to reconstruct faces were obtained in two samples. One was the sample of skulls belonging to solved forensic cases that were acquired from Sri Jayewardenepura and Karapitiya Medical Faculties.Photographs of the each of the deceased were also acquired to be compared with the reconstructed final 3D face.The second sample was CT scan patient data. Scan data of patients who had come for head scans were used with the permission from hospital authority and a DICOM viewing software (CT scan image viewing software) was used to extract a the skull and the patient's actual face via the $3 \mathrm{D}$ surface rendering function of the editor for evaluation purpose.

Ideally when an unknown skull is found, the following process will have to be carried out, with a pre-requisite stage and four main steps as follows.

Pre-requisite: Forensic Analysis of the Skull of the deceased Person

Step 01 : Capturing the 3D model of the skull

Step 02 : Placement of Landmarks

Step 03 : Digitally Sculpting the Face

Step 04 : Adding Facial Components

\section{Pre-requisite: Forensic Analysis of the Skull}

This stage was performed when no prior knowledge on the owner of the skull was present, which would be the case in any unidentifiable corpse. And in such instances, this analysis was performed to determine certain information about the deceased person from the skeletal remains of the corpse.A forensic medical expert extracted the following details from the skeletal remains.

- General age category (Normally an assumption in a bracket of 5-10 years would suffice)

E.g. 25-30 years

- Sex

These identified features assisted the reconstruction process in step 02 .

\section{A. Step 01: Capturing the Skull}

As the first step, the 3D model of the skull was acquired. Out of the two schools of thought that were mentioned in the related work, using the skull as the sole base of the reconstruction technique was used by the researchers, and hence acquiring a fairly accurate model of the skull was of paramount importance.

To capture the real life skull samples the Vivid $9103 \mathrm{D}$ scanner of Konica Minolta ${ }^{\mathrm{TM}}$ (Figure 10) was used. This was mainly due to the reason that it was already been used to generate models of human components in the Medical Field.

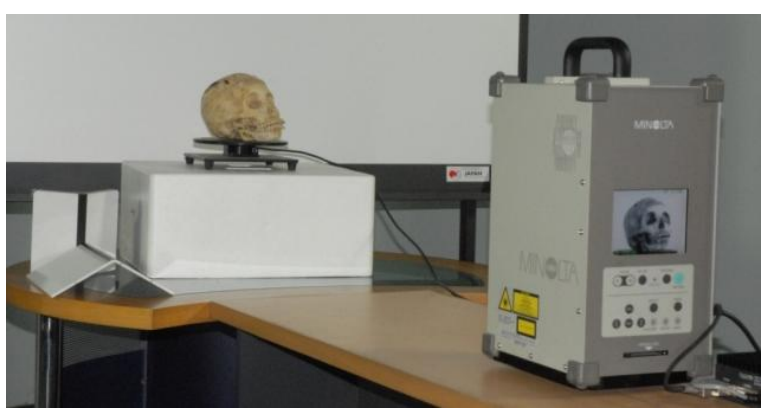

Fig. 10Our set up of acquiring the3D model of the skull with the Vivid 910 3D Laser scanner at University of Colombo

Once the 3D models were acquired using the 3D Scanner, it was observed that most of the test skulls were fractured and damaged. Nevertheless this was not an unexpected dilemma since most the human remains unearthed after years would be in a dilapidated stage.

However, this status of the skulls rendered it hard to conduct an accurate reconstruction. Hence it was required to make certain rectifications to the $3 \mathrm{D}$ models of the skulls. A $3 \mathrm{D}$ editing software application was utilized in this stage to successfully rectify the acquired 3D models. (Figure $11[\mathrm{a}, \mathrm{b}]$ ).

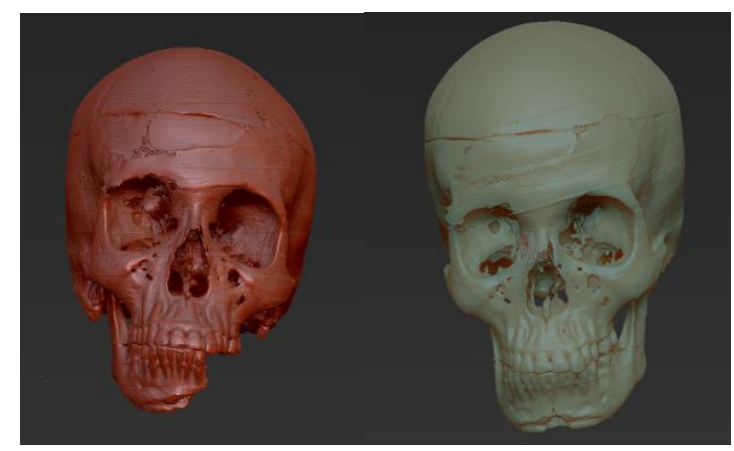

Fig. 11 (a) Test skull with half of the jaw missing (b) After applying the symmetry modifier in a 3D editing software

\section{B. Placing Landmarks}

Once the 3D model of the skull was obtained, the next step was to define anatomic and physiognomic points, known as landmarks, on the 3D model of the skull (Figure 12). Other studies on facial reconstructions have each used a differing number of landmarks, andhence the number of landmarks the researchers of this study used was optimized to suit in to their method.

The length of each of these landmarks was determined by the soft tissue thickness of the facial area in which the landmark was placed. Since such data was not available on Sri Lankans, an independent research on the facial soft tissue thickness of the Sri Lankan people was also carried out by the researchers using personal computer based Magnetic Resonance Imaging (MRI) and Computed Tomography (CT) images and its results are mentioned in section IV, Analysis Results.

In the course of the research it was found that the facial tissue thickness of a person differs based on age, sex, weight and race. Yet when Sri Lanka is taken in to consideration, all Sri Lankans can be classified in to the broad category of Caucasoid. Therefore in measuring tissue thickness data, the race category was exempted but data was gathered based on the age, sex and weight categories. 


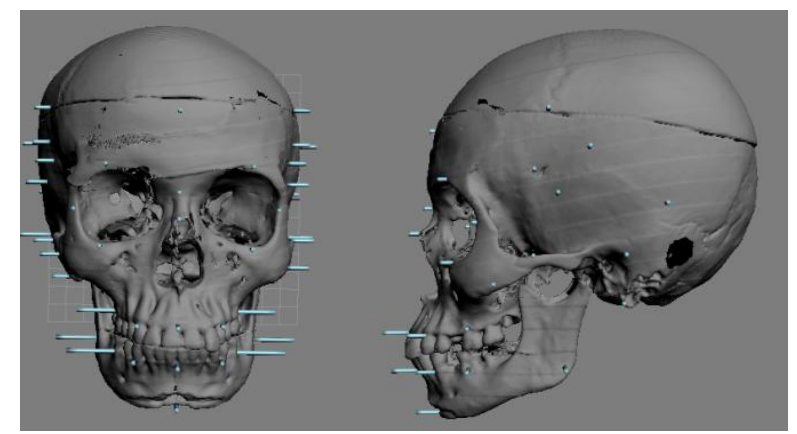

Fig. 12The skull model with the tissue thickness landmarks (Case 01)

For this study, tissue thickness data obtained from a sample of people who were similar to the deceased in terms of Age and Sex (as identified in the pre-requisite stage) was considered.

For instance, in the first case study it was assumed that the deceased was a medium weight female of the age category 2030. Thus the tissue thickness data gathered people belonging to those specifications were used. And by using MRI and CT images of the sample group, tissue thickness depths for each of those anatomical landmarks (Figure 13) were measured. Head/ Sinus scans (without the patient identification details) obtained from medical institutions were used for this purpose.

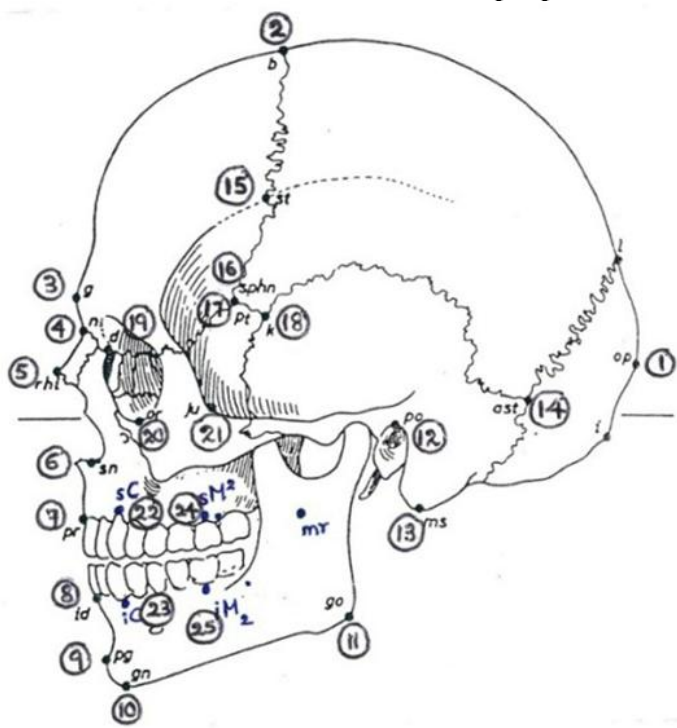

Fig. 13Lateral view of the skull showing landmarks from 1-25

By using those depth values, the height of the tissue thickness landmarks placed on the model were then adjusted. (Note: This study was carried out as a proof of concept for the people belonging to the Medium weight category. Hence forensic cases where the deceased was of a medium weight were chosen. Therefore tissue thicknesses were also gathered from a sample of people belonging to the medium weight category).

\section{Digitally Sculpting the Face}

As with all the other human organs, the face is also constructed with a complex structure of muscles that forms the basis of the skin layer. The method that was used in the research was to reconstruct these muscles on the skull and to generate the skin based on those muscles.

Several techniques were tried out and it was decided to use the $\mathrm{ZBrush}^{\mathrm{TM}}$ software for the muscle reconstruction. This was due to the reason that it was digital sculpting software hence there would be a direct map between the manual reconstruction (sculpting) and the usage of this technique. Based on the knowledge gathered on the human anatomy (Figure 14), the muscles were constructed.

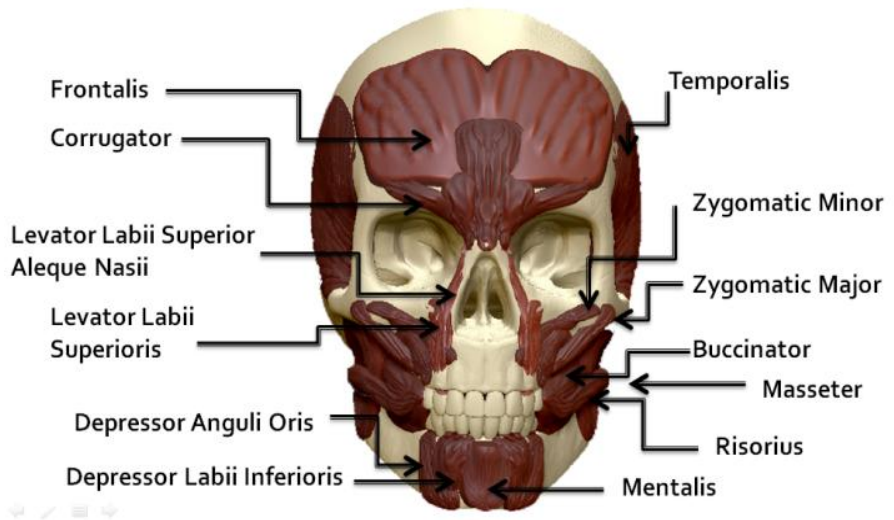

Fig. 14The initial stage of the skull after applying muscles based on human facial anatomy

The thicknesses of each of these muscles were determined by the landmarks placed in the earlier stage. Each muscle was "inflated" or "trimmed", "polished" until the thickness specified by the landmarks was achieved.

After the face was reconstructed, the addition of different facial components based on user requirement (broad, narrow noses) was incorporated at a later stage in the research. Yet, some basic foundation had to be laid on the face in order to add different components later. Therefore, the construction of the basic muscles was followed by the construction of more complex facial components such as eyes and nose in a basic level.

The dilemma faced in constructing these components was that all the facial components were supposedly created from cartilages. Therefore unlike the other muscles, the bone structure could not be used as the sole basis for these constructions. Hence after applying the base muscles the application, then facilitated the reconstructor to adopt the following guidelines to predict the placement of facial components.

a) Eyes: $25 \mathrm{~mm}$ diameter eyeballs [17] were placed in the orbits, and positioned so that the eyeball and pupil were centrally located within the orbits [18][19]. The eyeballs were positioned in the orbit so that a tangent taken from the superior to the inferior mid-orbital margins touched the iris (Fig. 15(a)). Eyeball protrusion was determined using the following formulae [20]

$$
\text { Eyeball protrusion }=18.3-(0.4 * \text { orbit depth })
$$

b) Nose:The maximum width of the soft nose was estimated by the bony nasal aperture at its widest point as three-fifths of the overall width of the soft nose (Figure 15(b)) [21].
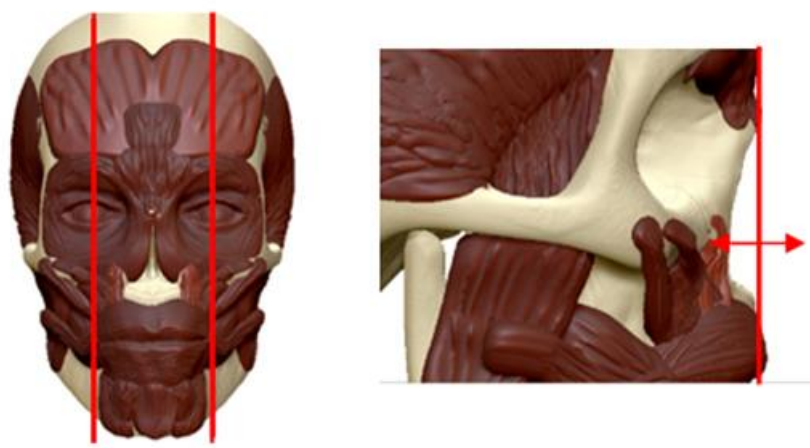

Fig. 15(a)The lip position based on the eye ball (b) The nose position based on nasal cavity 
c) Mouth:The corners of the mouth were estimated by the maxillary canine and first premolar teeth[19][22]. The following formulae was adopted since Sri Lankans fall in to the category of Asians from the Indian subcontinent according to [23]

Upper lip thickness $=3.4+0.4 \times$ (upper incisor height)

Lower lip thickness $=6+0.5 \times$ (lower incisor height)

Total lip thickness $=7.2+0.6 \times$ (upper and lower incisors height)

d) Ears:The broad length of the ears was predicted by the length of the nose [24]; the ear canal was positioned using the external auditory meatus [19]. The ear angle was set parallel to the jaw line; earlobe adherence was predicted by direction of the mastoid processes[21]

These steps created a several steps stages? (Figure 16) through which the final face was reconstructed.
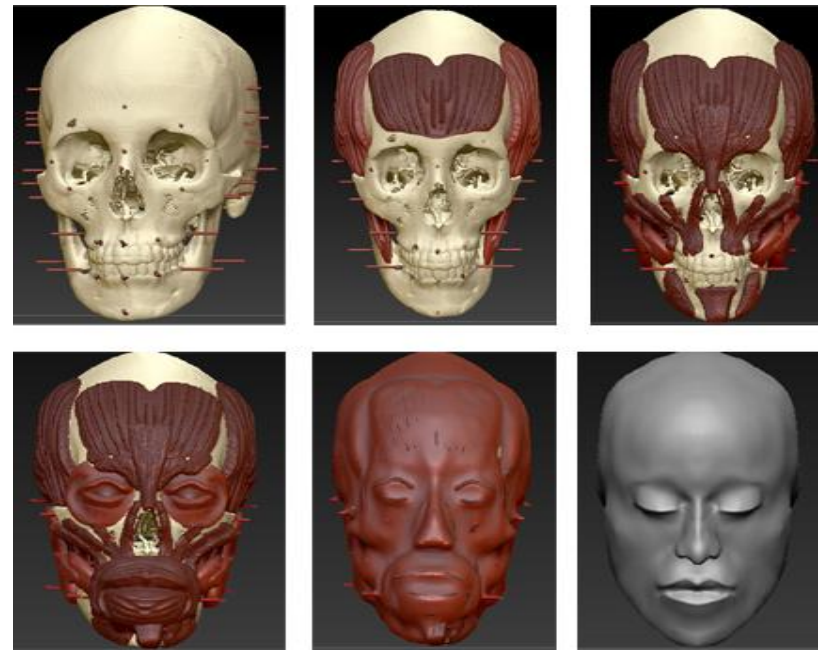

Fig. 16The different stages of the reconstruction procedure (Case 02- Male/2030)

The final step in face building was to apply skin on top of all the muscles. A technique called "remesh all" facilitated this function in ZBrush ${ }^{\mathrm{TM}}$. After the skin was applied, the face was edited to get the underlying muscle effects and the structure, using tools available in ZBrush ${ }^{\mathrm{TM}}$ (Figure 17).

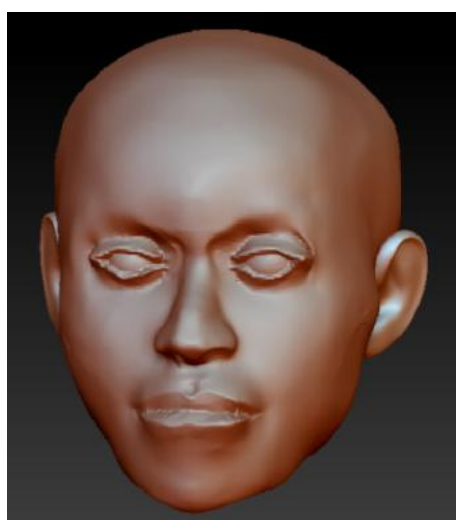

Fig. 17The Intermediate model created using the muscular structure (Case 01)

\section{Adding Facial Components}

A realistic appearance for the reconstructed face was to be obtained by modelling the facial features such as eyes and noseappropriately. Yet, data on different types of eyes and noses was not available since such an analysis had not been conducted on Sri Lankans so far. Therefore, a separate facial feature analysis was carried out on Sri Lankans and the result of this analysis is in the analysis results section.

Based on the analysis the most common dimensions of the facial features were discovered and by using these values, facial components (different sizes and shapes) of the highest frequency among the target group were added to the 3D model to improve the possibility of identification. (Refer Table I, II in Analysis Results section).

Even though eyes and nose were added to the face (Figure 18), adding different hair styles for the reconstructed face was disregarded since it tends to change the appearance of the final outcome drastically which might also render an output unidentifiable to the deceased person's relatives.

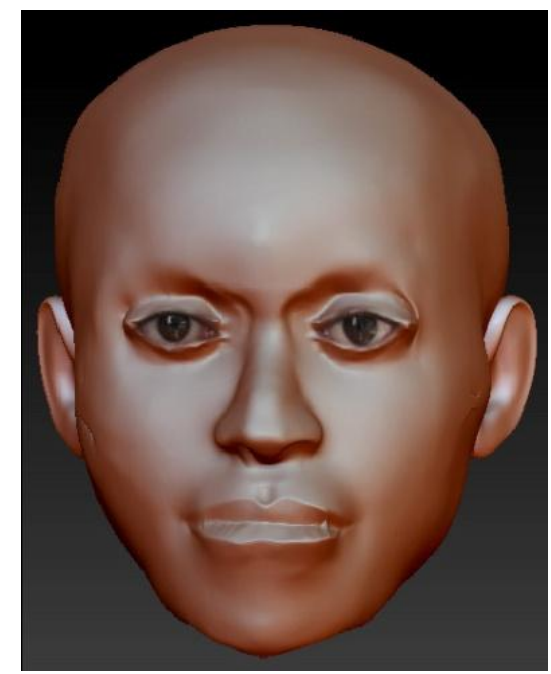

Fig. 18The Final model of the process (Case 01)

The addition of different combinations of facial features on to the reconstructed face resulted in generating a considerable amount of different facial models, which could subsequently increase the percentage of the identification of the deceased.

\section{RESULTS}

\section{A. Analysis Results}

1) Tissue Thickness Analysis

The sample used for measuring the facial soft tissue thickness consisted of Sri Lankan Females ranging in age from 20 to 30 years of age and in the medium weight category.Brain MRIs and CT scans of the selected group were included to acquire tissue thicknesses.

The soft tissue thickness of 30 points were measured for this study and out of those 30 points 17 points were selectedfor the comparison of statistical data published by different authors for North West Indians, American blacks (African Americans),American Whites (American Caucasians)and for Japanese.

When selecting the Brain MRIs for measuring the facial tissue thicknesses, it was determined to select MRIs of people who do not have head and neck trauma or any other pathology because such pathology may sometimes result in disfiguring the normal facial structures. Such MRIs were excluded from the study.

The results of the measured soft tissue thicknesses of Sri Lankan female are tabulated in the Table 1. Mean values for tissue thicknesses are presented in millimeters. 
TABLE I

FACIAL TISSUE DEPTH DATA FOR SRI LANKAN FEMALES AGED 20-30

\begin{tabular}{|l|l|}
\hline $\begin{array}{l}\text { Point Number/Name of } \\
\text { the Landmark }\end{array}$ & Mean Values (mm) \\
\hline 1.Opisthocranion & 5.87 \\
\hline 2. & 5.49 \\
\hline 3.Glabella & 3.25 \\
\hline 4.Nasion & 5.32 \\
\hline 5.End of Nasal bone & 3.22 \\
\hline 6.Mid-philtrum & 10.58 \\
\hline 7.Upper lip margin & 10.02 \\
\hline 8.Lower lip margin & 10.2 \\
\hline 9.Mental eminence & 9.41 \\
\hline 10.Beneath Chin & 17.08 \\
\hline 11.Gonion & 23.98 \\
\hline 12.Supra glenoid & 10.95 \\
\hline 13.Mastoidale & 8.75 \\
\hline 14.Asterion & 7.84 \\
\hline 15.Stephanion & 5.77 \\
\hline 16.Sphenion & 7.91 \\
\hline 17.Pterion & 10.23 \\
\hline 18.Krotaphion & 10.48 \\
\hline 19.Supra orbital & 5.5 \\
\hline 20.Infra orbitale & 6.35 \\
\hline 21.Jugale & 9.66 \\
\hline 22.supra-Canine & 8.33 \\
\hline 23.infra-Canine & 19.87 \\
\hline 24.Supra M2 & 25.2 \\
\hline 25.Sub M2 & 23.4 \\
\hline 26.Euryon & 8.03 \\
\hline 27.Inferior malar & 12.8 \\
\hline 28.ectocochion & 3.37 \\
\hline 29.Zygomatic arch & 9.33 \\
\hline 30.Maxillofrontale & 9.03 \\
\hline 31.Occlusal line & 20.23 \\
\hline
\end{tabular}

After the tissue thickness analysis, it was found that a considerable difference exists between the facial soft tissue thicknesses of Sri Lankans and other races? Nationalities? (not sure check). Hence applying tissue thickness values of other countries to a Sri Lankan skull model would result in inaccurate approximations.

During the comparisons it was also observed that Sri Lankans have differing facial soft tissue thickness depths at the areas of beneath Chin, Gonion, Sub M2 (Below the second mandibular molar) and Supra M2 (Above the second maxillary molar).

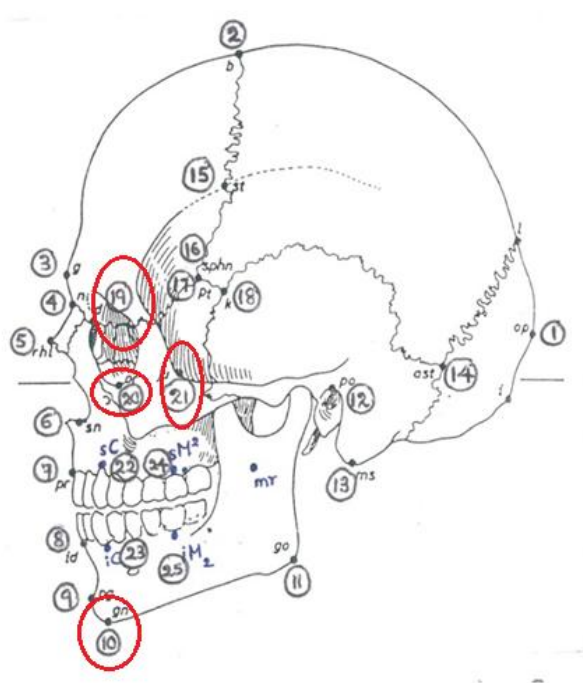

Fig. 19Areas of differing tissue thickness in Sri Lankans as compared with the data in other regions

\section{2) Facial Feature Analysis}

The facial feature analysis resulted in the most common nasal and eye indexes. For this analysis a sample of 426 (290 males and 136 females) of the age category 20-30 was used and the sizes and shapes of the most commonly occurring facial components were devised. In obtaining the measurements of each facial component a photo editing and measuring software was used and in it, the human error rate was also calculated to improve the accuracy of measurements.

a) Nose Analysis: Using the photographs of the sample group the nasal index was calculated using the following formula.

$$
\text { Nasal Index }=\underline{\text { Nose width }} * 100
$$

Nose height

After which the indexes were categorized as narrow medium and broad and the most frequent N.I for each of the categories were devised. (Table II)

TABLE II

THE MOST FREQUENT NASAL INDEX

\begin{tabular}{|l|l|l|}
\hline $\begin{array}{l}\text { Nose } \\
\text { Categories }\end{array}$ & $\begin{array}{l}\text { Most frequent N.I } \\
\text { (Male) }\end{array}$ & $\begin{array}{l}\text { Most frequent N.I } \\
\text { (Female) }\end{array}$ \\
\hline Narrow & 100 & 80 \\
\hline Medium & 103 & 100 \\
\hline Wide & 136 & 104 \\
\hline
\end{tabular}

b) Eye Analysis: Same technique was followed to measure variations in the eyes. (Table III)

Eye Index $=$ Eye width $* 100$

Eye height

TABLE IIITHE MOST FREQUENT EYE INDEX

\begin{tabular}{|l|l|l|}
\hline $\begin{array}{l}\text { Eye } \\
\text { Categories }\end{array}$ & $\begin{array}{l}\text { Most frequent E.I( } \\
\text { Male) }\end{array}$ & $\begin{array}{l}\text { Most frequent E.I } \\
\text { (Female) }\end{array}$ \\
\hline Narrow & 300 & 257 \\
\hline Medium & 355 & 300 \\
\hline Broad & 733 & 400 \\
\hline
\end{tabular}

\section{B. Evaluation Results}

The forensic facial reconstructions are conducted with the ultimate intention of constructing a face that will be identifiable by the deceased person's relatives. Hence a reconstruction has to be mainly identifiable andhave a resemblance to the deceased. Two methods of evaluations were conducted to evaluate these features in the reconstructed faces, one for real life skulls sample other for CT scan patient sample.

- Resemblance Rating System

- Face pool Comparison

The reconstructed face has to be similar to the deceased. This feature was evaluated using another two methods of evaluations.

- Photogrammetric Analysis

- Morphing Software to Superimpose

1) Resemblance Rating System

The CT scan patient sample was evaluated under this method and an online survey was used for it. 


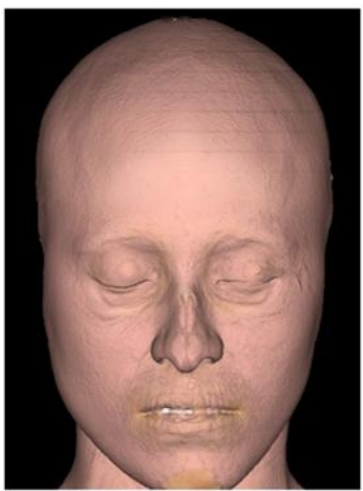

Actual Photograph of the Patient

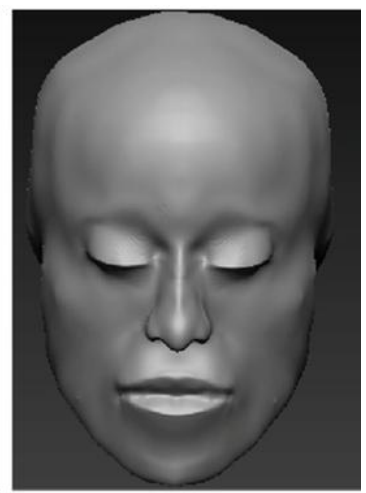

Reconstructed 3D model of the face
Fig. 20Online Survey created for Resemblance Rating System

The reconstructed face was placed alongside the 3D face given by the CT image editing software and the respondents were asked to rank the resemblance of the two on a scale of 0100. Results obtained for some cases were as follows.

TABLE III

SOME RESULTS OF THE RESEMBLANCE RATING SYSTEM EVALUATION

\begin{tabular}{|l|l|l|l|l|}
\hline \multirow{2}{*}{$\begin{array}{l}\text { Case } \\
\text { Number }\end{array}$} & \multicolumn{4}{|c|}{ Similarity } \\
\cline { 2 - 5 } Overall Face & Outer Contour & Mouth & Nose \\
\hline Case 3 & $97 \%$ & $92 \%$ & $45 \%$ & $100 \%$ \\
\hline Case 4 & $89 \%$ & $79 \%$ & $91 \%$ & $98 \%$ \\
\hline
\end{tabular}

\section{2) Face Pool Comparison}

This evaluation method was conducted for the sample of real life skulls that had the deceased persons photograph. An online survey was conducted where respondents were requested to select the photograph that is most similar to the reconstructed $3 \mathrm{D}$ model, out of a range of photos which included the diseased person's actual photo as well.

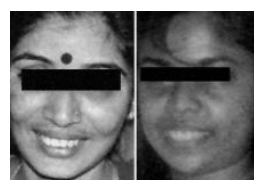

1

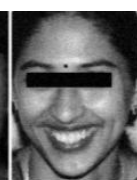

3

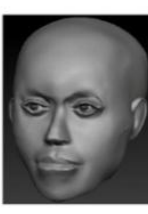

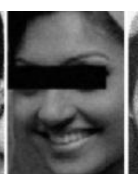

4

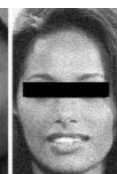

5

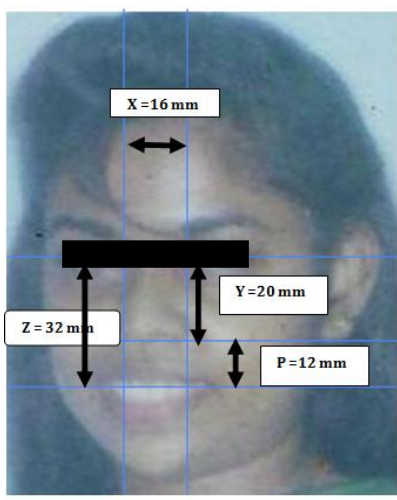

Actual Image (A1)
TABLE V

THE RESULTS OF FACE POOL COMPARISON

\begin{tabular}{|l|l|l|l|l|l|}
\hline $\begin{array}{l}\text { Case } \\
\text { Number }\end{array}$ & Photo 1 & Photo 2 & Photo 3 & Photo 4 & $\begin{array}{l}\text { Real } \\
\text { Photo }\end{array}$ \\
\hline Case 1 & $14 \%$ & $9 \%$ & $6 \%$ & $19 \%$ & $52 \%$ \\
\hline Case 2 & $7 \%$ & $15 \%$ & $12 \%$ & $22 \%$ & $44 \%$ \\
\hline
\end{tabular}

\section{3) Photogrammetric Analysis}

This evaluation method was adopted to measure the similarity between the reconstructed and real faces. In this, the distance between specific facial components in the model and the actual photograph were measured and compared. The chosen components were the standard components that are always used in the photogrammetric analysis. The logic behind this technique was that if the reconstruction process was of high accuracy the difference ratio between the facial components of the real life image and the modelled image would be less.

Fig. 22Photogrammetric Analysis

In figure $22, \mathrm{X}$ refers to the distance between the starting point of two eyes closest to the nose, $Y$ refers to the distance between that same point in a eye to the end point of nose, $\mathrm{Z}$ refers to the distance between eye and mouth and finally $\mathrm{P}$ refers to the distance between mouth and nose. The results acquired by measuring the distance between these components in some of the reconstructed faces are in the table VI.

TABLE VI

RESUlTS OF THE PHOTOGRAMMETRIC ANALYSIS

*Unable to obtain clear values

\begin{tabular}{|l|l|l|l|l|}
\hline $\begin{array}{l}\text { Case } \\
\text { Number }\end{array}$ & $\mathbf{X}$ & $\mathbf{Y}$ & $\mathbf{P}$ & $\mathbf{Z}$ \\
\hline Case 1 & $0 \%$ & $10 \%$ & $8.33 \%$ & $3.125 \%$ \\
\hline Case 2 & $3.33 \%$ & $*$ & $*$ & $8.78 \%$ \\
\hline Case 3 & $0 \%$ & $10 \%$ & $10 \%$ & $3.33 \%$ \\
\hline Case 4 & $14.28 \%$ & $3.44 \%$ & $18.18 \%$ & $25 \%$ \\
\hline
\end{tabular}

\section{4) Morphing Technique}

Another evaluation was done using free morphing software which is known as Free Morphing 2.1. The software was used to identify the differences existing between the ante mortem photograph of the deceased (source image) and the 3D model reconstructed (target image). This method is closely related with superimposition technique which is commonly used to identify missing people when an ante mortem photograph of the deceased is present. 
The image sequence (Figure 23) generated by the software for a given source and the target image clearly shows how the facial features and the contours of the face, morphs from one to another. By identifying the intermediary transformation stages, the authors were able to verify the soft tissue alignment and to observe if there were any obvious errors.
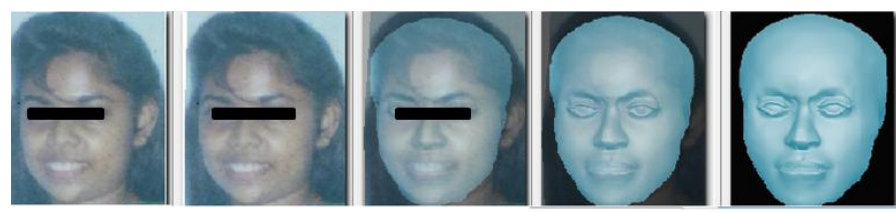

Fig. 23.Image Sequence Generated by the Morphing Software for the given source image and the target image

Thus it is evident that all the evaluation methods adopted to measure the identifiability and similarity resulted in positive feedback.

\section{DISCUSSION}

\section{A. Challenges Faced}

As a research that explored an area that had hitherto not been addressed by any researcher in Sri Lanka, this research had resulted in testing researchers' perseverance and commitment in many stages. The scope of research had to be kept strictly in mind since most of the pre-requisites of the research were not fulfilled by other researchers in that area.

For the facial tissue thickness analysis data had to be gathered from several private and governmental hospitals where the researchers had to spend time getting familiarized with the CT and MRI scanning machines, access to which wasn't granted easily.

Once access was granted to the tissue thickness data, it was found out that most of the patients who apply for brain scans were between the age ranges of 40-60. And yet the test skulls the forensic officers had been able to supply the researchers with were all from the age category of 20-30. Hence, it had required extensive effort from researchers to gather tissue thickness data belonging to this group. Another challenge they had faced with was, the manner brain scans were conducted. In order to have minimal effect to the patients from the rays emitted during a scan, most of the brain scans had not included a complete head scan, and hence the Mandible (jaw bone) was missing from most of them. Therefore researchers had to search for brain scan data that included the Mandible, in all the hospitals they had access to.

In obtaining photographs for facial feature analysis and tissue thickness data, the researchers were hassled by ethical issues as well. In order to overcome this predicament, consent was obtained from each individual to use their photograph for research purposes and since the tissue thickness data didn't involve any patients' or patient's? personal information, permission was granted by hospital administrations to use the data for the research purpose.

\section{B. Alternative Input Mechanism}

Since 3D scanners are not commonly available, it was found that using CT images of the skull can also be used as a viable option to generate $3 \mathrm{~d}$ models. The advantage of this method is that since the CT scanner possesses the capability to capture details precisely, the resulting model would be of very high accuracy and detail. The DICOM output of the CT scanner was imported in to an image processing application dedicated to DICOM images [Osirix-viewer] and the 3D model was obtained.

\section{Importance of Used Semi-Automated Methodology}

The proposed semi-automated methodology of reconstructing based on facial muscles; especially in the field of computer based 3D reconstruction is a novel approach.

The literature stated that the manual reconstruction method resulted in a natural looking output but lacked in efficiency and expertise. Further it was found from the course of this research that a fully automated technique would be more efficient compared to the manual technique. But the output due to the process being fully automated resulted in an unrealistic final model. Thus it was decided to use a semi-automated technique by combining the advantages of both the manual and fully automated techniques. This methodology automates the steps of the reconstruction process where inefficiencies occurred in the manual process(E.g. Creating a plasticine mould of the skull, Sculpting muscles manually using modelling clay). Since the manual reconstruction is done mostly by modelling the facial muscles on a model of the skull, the authors were of the opinion that having a direct map between this technique and the computerized 3D reconstruction process would result in a more natural looking output. Thus muscles were sculpted manually in a digital environment which unlike in the fully automated process, resulted in a more natural realistic output.

\section{Importance of the Solution}

Since all four evaluations gained positive results as well, reconstructing the face using the muscle based anatomy approach was proven to be reliable and accurate to a sufficient extent. The following problems were addressed by this research.

TABLE VII

SPECIFIC PROBLEMS ADDRESSED BY THIS RESEARCH

\begin{tabular}{|l|l|}
\hline Problem & Solution \\
\hline $\begin{array}{l}\text { Lack of Tissue thickness data of } \\
\text { Sri Lankans }\end{array}$ & Tissue Thickness Analysis \\
\hline $\begin{array}{l}\text { Lack of facial features differences } \\
\text { data on Sri Lankans }\end{array}$ & Facial Feature Analysis \\
\hline $\begin{array}{l}\text { Expert technical knowledge on } \\
\text { facial reconstruction process }\end{array}$ & $\begin{array}{l}\text { 3D sculpting based simplified } \\
\text { process }\end{array}$ \\
\hline High Cost of exiting solutions & $699 \$$ software cost \\
\hline Time Constraint & $4-5$ Hours \\
\hline
\end{tabular}

Once the cost analysis was carried out it was quite apparent that the solution presented was feasible especially in monetary terms. The cost of the software used, amounts to \$699 and that compared with the systems used in other countries is a very low cost solution. The time consumed was also considerably less when compared with the manual technique. Furthermore, the expertise in 3D modelling would not be an issue either due to the reason that to a 3D modelling artist the muscle sculpting learning curve would be substantially short. Comprehensive manuals and documentation have been created by the research team.

\section{E. Feedback from Other Parties}

In many unidentified dead bodies, the face has been rendered unrecognizable to the point of complete deterioration through autolysis, decay, by animals, or other destruction. In such situations, facial reconstruction can be used as a method of last resort.

According to Dr Wilkinson one of the world famous forensic anthropologists the computer aided forensic facial reconstruction is much more effective than manual technique. According to her;

"Today we can use information from 3D surface scans or CT scans of the skull, import them, and use 3D modeling or 'virtual 
sculpture' to create the same muscles that we would create in real clay"

According to forensic anthropologists adding the muscles over the skull gives the face its shape. Further, they state that ;"We all have the same muscles, but because every skull is a slightly different shape, each muscle is slightly different on each individual".

Most of the forensic anthropologists agree that one of the key advantages of the computer system is that they can turn layers on and off, switching from skin to the muscles or bone which is something that they can't do with the traditional methods because as soon as they put clay onto a skull it gets covered up from clay.

Another important thing is that, the technological means can be used to analyze delicate human remains without handling them. Once some skeletal remains were found Anthropologists do not need to remove the soft tissue to see the bone. They can analyze the skeletal remains with great care without harming the existing conditions. And also information can also be transferred quickly - scan data can be e-mailed from anywhere in the world, rather than having to transport skulls or casts of the bone. Due to these reasons computer aided reconstruction is considered as a more adaptable and flexible way of doing reconstruction.

The technology further allows forensic anthropologists to assess the accuracy of their work, because they can compare the reconstructed face models with the faces of living people. This feature helps a lot for the Judicial Medial Officers since this helps them to finalize cases that they are dealing with. Also this helps them since they can show the likely appearance of a missing person from a photograph taken many years ago. This will reduce the workload and improve the speed of their work.

However according to the anthropologists' reconstruction on the facial soft parts should be done with great care. Since all facial components such as eyes, nose and lips are missing from the skull, chances are high that reconstruction may give(produce) a false reconstruction which would lead to false identification. But according to forensic anthropologists different types of facial components could be added to a single reconstruction so that for the same skull a large number of face models could be reconstructed. This would increase the probability of the identification.

\section{F. Human Rights Aspect}

Due to the sensitivity of the subject matter ethical clearance is sought by the project team from the ethical review committee of the University of Colombo.

According to the forensic identification process, when a corpse is unable to be identified through the existing mechanisms such as DNA analysis, finger print analysis, etc., it will be buried thus resulting in its identity being a mystery forever. Even though many argue that the deceased do not posses human rights, it should be regarded that being buried in accordance with one's religion and traditions in a grave that bears one's own name, a human right. Thus with regard to unrecognizable corpses, its identification is of high importance and when all other identification mechanisms fail, this process of forensic facial reconstruction comes into picture.

\section{G. Future Work}

Several areas can be pointed out in to which this work can be carried forward. One such significant area is to incorporate this system to the National missing people database (is this a name? then keywords should be caps). If implemented, then the reconstructed faces could be compared with those in the database for a positive identification. The same technique of facial recognition which was used in the evaluation stage of this project could be used for this purpose.

This can also be extended to be used by the archaeologists. If a method could be determined to unearth the tissue thicknesses of the excavated human remains, the same framework that has been suggested by the researchers to rebuild the faces of modern man can be adopted to build the faces of our ancestors as well. And hence a realistic face could be given even to the Balangoda Man himself.

\section{H. Conclusion}

Although facial reconstruction using computer technologies is widely used in developed countries, it is still used at the prefatory level in Sri Lanka. And the aim of this research is to introduce these technologies to the forensic experts who are yet to move away from the manual clay based reconstruction techniques.

A substantial amount of researches have been carried out in the area of facial reconstruction, but reconstructing via the use of facial muscles, especially in the field of computer based 3D reconstruction are rare. Further, it aided to produce a more natural-looking face. Hence, reconstructing a face using a semiautomated muscle based anatomy approach is proven to be reliable and accurate.

Facial approximations for the 20-30 age group and medium weight cases were produced by this research. The outputs were successful and thoroughly evaluated using accepted evaluation techniques. For the process, optimized tissue thickness landmarks and an accepted facial muscle anatomy based model were used. Although the research was performed on certain age and weight groups, this process would be scalable to any other age/weight groups as well (given that the tissue thickness data for all those groups are collected.)

Thus it was concluded that implementing a national unit for facial reconstruction, which would adopt this procedure would be a very timely act for the island

\section{ACKNOWLEDGEMENT}

The medical officers at the Radiology unit and Dr. (Mrs) Gulpa Subasinghe, Consultant Radiologist, Central Hospital (Asiri Group), Colombo are reminded with gratefulness for their support extended regarding the tissue thickness research of Sri Lankans. And Dr.Vibash P. Wijeratne, Manager-Allied Medical Services, Durdans Group of Hospitals and Prof R. Fernando of the Colombo Medical faculty would be remembered with muchgratitude for their kind assistance.

\section{REFERENCES}

D. Collon, Ancient near Eastern art. London: British Museum Press, 1995, pp. 41-42.

[2] G. Watts, "Histories: The girl from the Seine," New Scientist, 2005

[3] C. Nyborg, "Oliver Cromwell's death mask at Warwick Castle.," 2006. [Online]. Available:

http://en.wikipedia.org/wiki/File:WarwickCastle_CromwellDeathmas kcrop.JPG. [Accessed: 03-Sep-2012].

[4] Wikipedia contributors, "Death Mask." [Online]. Available: http://en.wikipedia.org/wiki/Death_mask.

[5] P. Vanezis, R. W. Blowes, a D. Linney, a C. Tan, R. Richards, and R. Neave, "Application of 3-D computer graphics for facial reconstruction and comparison with sculpting techniques.," Forensic science international, vol. 42, no. 1-2. pp. 69-84, Jul-1989.

[6] C. M. Wilkinson, Forensic facial reconstruction. . Cambridge University Press, 2004.

[7] a. F. Abate, M. Nappi, S. Ricciardi, and G. Tortora, "FACES: 3D FAcial reConstruction from anciEnt Skulls using content based image retrieval," Journal of Visual Languages \& Computing, vol. 15, no. 5, pp. 373-389, Oct. 2004.

[8] B. Andersson and M. Valfridsson, "Digital 3D Facial Reconstruction Based on Computed Tomography Digital 3D Facial Reconstruction Based on Computed Tomography," 2005 
[9] M. Silvestri and G. Tomezzoli, "3D Facial Reconstruction from a Skull of a Male Subject of the Neolitic ' square mouth vases' Culture of Quinzano ( Verona-IT )," no. 3.

[10] P. Vanezi, M. Vanezis, G. McCombe, and T. Niblett, "Facial reconstruction using 3-D computer graphics.," Forensic science international, vol. 108, no. 2, pp. 81-95, Feb. 2000.

[11] G. M. Gordon and M. Steyn, "An investigation into the accuracy and reliability of skull-photo superimposition in a South African sample.," Forensic science international, vol. 216, no. 1-3, pp. 198.e1-6, Mar. 2012.

[12] P. Tu, R. I. Hartley, W. E. Lorensen, M. Allyassin, R. Gupta, and L. Heier, "Face Reconstructions using Flesh Deformation Modes," pp. $1-17$.

[13] S. De Greef and G. Willems, "Three-dimensional cranio-facial reconstruction in forensic identification: latest progress and new tendencies in the 21 st century.," Journal of forensic sciences, vol. 50 no. 1, pp. 12-7, Jan. 2005.

[14] R. Romeiro, R. Marroquim, and C. Esperanc, "Forensic Facial Reconstruction using HRBF."

[15] S. Davy, T. Gilbert, M. Evison, and D. Schofield, "Forensic facial reconstruction using computer modeling software," ComputerGraphic Facial Reconstruction, pp. 183-194, 2005.

[16] S. Miyasaka, M. Yoshinoa, K. Imaizumia, and S. Setab, "Forensic SCience The computer-aided facial reconstruction system," vol. 74, pp. 155-165, 1995.
[17] S. Tian, Y. Nishida, B. Isberg, and G. Lennerstrand, "MRI measurements of normal extraocular muscles and other orbital structures," Graefe's Archive for Clinical and Experimental Ophthalmology, vol. 48, no. 5, pp. 393-404, 2000.

[18] B.P. Gatliff, "Facial sculpture on the skull for identification," American Journal of Forensic Medicine and Pathology, vol. 5, no. 4, pp.327-332, 1984

[19] W.M. Krogman and M.Y. Iscan, The Human Skeleton in Forensic Medicine, $2^{\text {nd }}$ ed., Illinois, Springfield, III: Charles Thomas, 1986

[20] C.M. Wilkinson and S.A. Mautner, "Measurement of eyeball protrusion and its application in facial reconstruction," Journal of Forensic Sciences, vol. 48, no. 1, pp.12-16, 2003

[21] M.M. Gerasimov, The reconstruction of the face from the basic structure of the skull, Russia: Publishers unknown, 1955

[22] R.M. George, "Anatomical and artistic guidelines for forensic facial reconstruction," in Forensic Analysis of the Skull, M.H. Iscan, R.P. Helmer, Eds. Wiley-Liss, New York, pp. 215-227,1993

[23] C.M. Wilkinson, M. Motwani, and E. Chiang, "The relationship between the soft tissues and the skeletal detail of the mouth," Journal of Forensic Sciences, vol. 48, no. 4, pp.728-732, 2003

[24] B.A. Fedosyutkin and J.V. Nainys, "The relationship of skull morphology to facial Features," in Forensic Analysis of the Skull M.H. Iscan, R.P. Helmer, Eds. Wiley-Liss, New York, pp. 199213,1993 